\title{
Los diarios reflexivos en la tutoría clínica de enfermería. Significados atribuidos por los tutorados.*
}

\section{Reflective diaries in tutoring clinical the nursing. Meanings attributed by tutees}

Mtra. Sofía Rodríguez Jiménez•

Mtra. Margarita Cárdenas Jiménez.•

LEO. Anel Blando Palomino...

\section{Resumen}

El análisis de los diarios reflexivos forman parte de la investigación "La tutoría clínica como estrategia de enseñanza aprendizaje en Enfermería Fundamental" a través de la implementación de un Modelo de Tutoría Clínica Reflexiva donde los diarios reflexivos se aplican como estrategia de enseñanza aprendizaje e investigación

Objetivo: Identificar los significados que le asignan los tutorados a la práctica clínica de Fundamentos de Enfermería II, a través de los diarios en un modelo de tutoría clínica reflexiva. Metodología: Análisis del contenido y significado de los diarios reflexivos desde la interpretación cualitativa de los textos en tres etapas: codificación, categorización e interpretación y el establecimiento de los vínculos y relaciones en el proceso de categorización. ${ }^{1}$ El grupo de estudio lo integran catorce tutorados, tres tutoras clínicas y 138 registros durante cuarenta días. Resultados: Del análisis del discurso se derivaron las siguientes categorías: Temor vs confianza, percepción sobre las enfermeras, identidad profesional, el ser enfermera, percepción sobre la tutoría y relación entre la teoría y la práctica, mismas que dan cuenta de las reflexiones clínicas.

Discusión y conclusiones: Las categorías muestran las experiencias significativas que vive el tutorado en el entorno de práctica hospitalaria, favorecen el aprendizaje reflexivo y una relación humanizada entre el tutor-tutorado.

"Proyecto PAPIIT, No 31008-3, DGAPA-UNAM

-Responsable del Proyecto de Investigación y Profesor de Carrera Titular "A", T.C. ENEO-UNAM.

eneo_sofi@hotmail.com

..Corresponsable del Proyecto de Investigación y Profesor de Carrera Asociado "C", T.C. ENEO-UNAM.

mcardenasjimenez@gmail.com

...Becaria del Proyecto de Investigación, Licenciada en Enfermería y Obstetricia PAPIIT, DGAPA. UNAM.

ablando79@hotmail.com

FeCHA DE RECIBIDO: 31 MAYO 2012

FeCHA DE ENVIADO: 5 JUNIO 2012

FeCha de ACEPtación: 29 SEPTIEMbre 2012 


\section{Enfermería Universitaria}

Permiten individualizar la tutoría, la reflexión en la acción coloca en el centro del proceso al tutorado y tutor. Resultados: 96\% tuvo riesgo medio para la edad (15 a 19 años); aunque 9 tuvieron mayor riesgo debido a tenían 15 años o menos; 27\% eran solteras,23\%correspondían a un nivel socioeconómico bajo, dos terceras partes reportó una escolaridad de 9 años o menos. Respecto a los factores biológicos cuatro de cada 10 tuvieron riesgo medio o alto en función de su peso (35\% entre 41 a 50 kgs. y $6.1 \%$ con menos de 40 kgs); la gran mayoría eran nuliparas; $12 \%$ con tabaquismo positivo; $16 \%$ tuvo amenaza de aborto; $11 \%$ con amenaza de parto pretermino; el problema de salud más importante fue la infección de vías urinarias $28 \%$ la tenía controlada y 10\% activa. Discusión y Conclusión: Los resultados coinciden con otras investigaciones, pero el número de embarazadas de 15 años o menos es superior a la media nacional que es de 6 en mil; el perfil obstétrico es en mayor medida de mediano riesgo; falta conocer los riesgos y daños en el posparto y recién nacido para completar este perfil.

Abstract

Key

Words:

Reflective diary,

clinical experience,

clinical tutoring.

\section{INTRODUCCIÓN}

El diario reflexivo es una técnica de aprendizaje creativa, a través de la cual los tutorados tienen la oportunidad de efectuar una reflexión cotidiana sobre su forma de actuar, la de sus compañeros y la del tutor, así también sirve como guía en la autoevaluación del propio tutor cuando es este el que los realiza.
El principio orientador para el uso del diario está en el hecho de que el proceso de narrar la propia experiencia permite al tutorado reconstruir su historia y asignarle nuevos sentidos, se crea una relación dialéctica entre experiencia vivida y narrativa, Cunha ${ }^{2}$ refiere que cuando la persona organiza sus ideas para el relato construye su experiencia de manera reflexiva y eso puede ofrecerle nuevas bases para la comprensión de la práctica. Al narrar sus experiencias, las personas parten de sus creencias, expectativas y al hablar del pasado, consiguen anunciar nuevas posibilidades e intenciones. 
Los diarios propician una fuerte conexión entre experiencia vivida y reflexión respecto de la primera, las narrativas se presentan como herramientas que permiten el acceso al pensamiento, fijando la acción en el contexto en que ella ocurre y deja explícitas las comprensiones del sujeto, en un movimiento que permite volver a la experiencia. Eso favorece la continuidad, elemento esencial para el aprendizaje y para la producción del conocimiento. Con respecto a la segunda; reflexionar implica un patrón cíclico de deliberación, implica meditar sobre la experiencia, ya sea antes, durante y posterior al momento de la escritura.

Los diarios reflexivos contienen observaciones, sentimientos, reacciones, interpretaciones, reflexiones, pensamientos, hipótesis y explicaciones, ${ }^{3}$ al mismo tiempo permiten minimizar el sesgo de la subjetividad del investigador e incrementar la confiabilidad de la investigación. ${ }^{4}$

Se considera que esta técnica de aprendizaje si bien tiene como fin explícito -favorecer el aprendizajetambién facilita procesos introspectivos íntimos del tutorado, un refugio emocional ético, principalmente cuando se abordan temas sensibles de alto contenido emocional y afectivo como son las diálogos internos del tutorado en el entorno de la práctica clínica. Así mismo es considerado como un instrumento de evaluación formativa en donde el estudiante es el eje del proceso de aprendizaje.

En el proceso formativo de la enfermería el diario reflexivo ofrece las siguientes ventajas:

- El ejercicio personal de la reflexión promueve la profundización y la fundamentación de las respuestas humanas a problemas que en principio pueden parecer similares.

- Una profesión basada en la práctica, no es sinónimo de profesión rutinaria y mecánica. El aprendizaje a partir de la práctica requiere analizar los elementos que la componen, las secuencias y pasos en cada proceso y la variabilidad de los resultados.

- El desarrollo de habilidades, expresión de sentimientos, pensamientos y en general el desarrollo de valores que favorecen el proceso de comunicación, benefician la relación de ayuda que debe establecerse con la persona sujeto de cuidado. ${ }^{5}$

En este contexto, la reflexión individual no es suficiente para la promoción del desarrollo profesional, se requiere de la participación del tutor para que pueda ayudar a direccionar ese proceso, ofreciendo parámetros para la reflexión y confrontando a los tutorados para que el proceso de reflexión acerca de la práctica pueda ser profundizado y el aprendizaje sea significativo.

La tutoría como proceso permanente que se da a través de la interacción entre el tutor, el tutorado y el entorno social de aprendizaje involucra factores como: la historia de vida de los participantes, su contexto social y cultural así como las situaciones sociales que caracterizan el entorno institucional donde se realizan las prácticas clínicas, es a lo que Bourdieu denomina "habitus". Ciertamente, el habitus puede entenderse como un sistema de disposiciones adquiridas, permanentes y transferibles, que generan y clasifican acciones, percepciones, sentimientos y pensamientos en los agentes sociales (tutor-tutorado) de una cierta manera, generalmente escapan a la conciencia y a la voluntad. ${ }^{6}$

En la especificidad de la enseñanza de la enfermería en entornos clínicos, la tutoría clínica se concibe como "Un proceso de acompañamiento colaborativo a través de la atención personalizada a un alumno o un grupo reducido, por

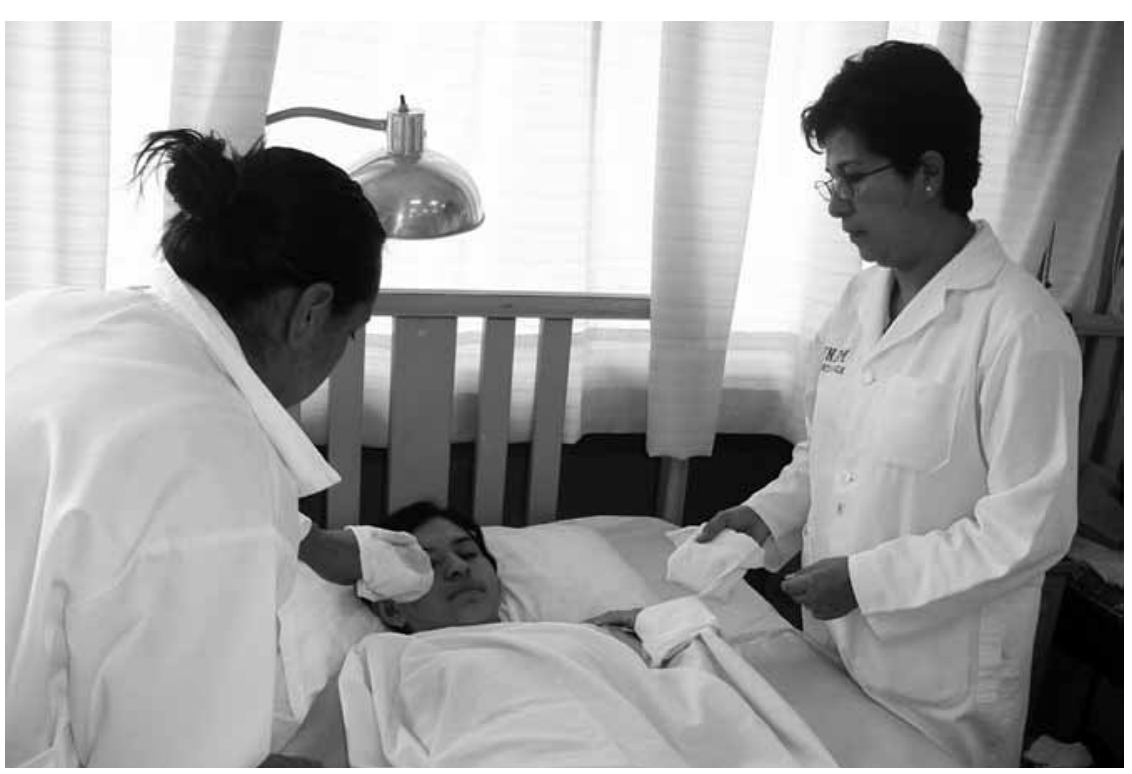


académicos expertos basados en el aprendizaje práctico, estrategias pedagógicas reflexivas y una comunicación dialógica, que permita entender las necesidades de los tutorados para su formación profesional integral, donde éstos y el tutor se comprometen en el aprendizaje del cuidado". 3 Se orienta hacia tres dimensiones: la personal, la académica y la profesional.

La dimensión personal reconoce que los tutorados son personas únicas en su estilo de aprendizaje y motivación, se individualiza la tutoría clínica conforme los intereses y necesidades del tutorado y del entorno profesional, adquiere sentido el trabajo colaborativo entre el tutor y tutorado y el desarrollo de habilidades para el desarrollo de habilidades para la reflexión y la argumentación entre otras.

La dimensión académica reconoce el contexto curricular específico, los objetivos y actividades del área de formación, así como las habilidades para el aprendizaje autónomo.

La dimensión profesional es el hilo conductor de la formación del tutorado para el desarrollo de los conocimientos, valores, actitudes y habilidades necesarias para el logro del perfil profesional y consolidación del ser profesional. ${ }^{7}$

En síntesis, a través de los diarios reflexivos se pretende que cada tutorado se permita dudar de aquellas concepciones que siempre ha considerado como verdades absolutas y que de alguna manera han limitado su forma de pensar y actuar, sus experiencias y aprendizajes anteriores, para analizarlos e integrarlos en una nueva construcción de su saber y reflexionar en torno a su proceso de aprendizaje, pudiendo así desenvolverse como un profesional reflexivo en un futuro. ${ }^{8}$

Objetivo: Identificar los significados que le asignan los tutorados a la práctica clínica de Fundamentos de Enfermería ll, en una unidad hospitalaria a través de los diarios reflexivos.

Metodología: Los diarios reflexivos se escribieron a partir de las experiencias vividas por los tutorados durante el periodo de prácticas hospitalarias, éstas se realizaron en un hospital público de tercer nivel de atención en el Distrito Federal, los servicios seleccionados fueron: Cirugía general, Medicina interna de Hombres, Medicina interna de Mujeres, Urgencias, Central de Equipos y Esterilización, Cardiología, Oftalmología y Otorrinolaringología.

Los tutorados fueron coordinados además de las tres tutoras Investigadoras, por la coordinadora de enseñanza de enfermería del hospital quién les dio la bienvenida e hizo la presentación de los mismos con las Jefes de servicio y las enfermeras y auxiliares de enfermería.

Los tutorados se insertaron al sistema de organización establecido en la institución en equipos de profesionales conformados por una enfermera general y una auxiliar de enfermería y una estudiante quienes eran responsables de ofrecer cuidados a un promedio de nueve personas hospitalizadas.

La población cuidada se constituyó por adultos jóvenes y adultos mayores.

Se trató de un estudio de investigación-acción, cualitativo, a través del análisis de textos plasmados en los diarios reflexivos, es- tos se escribieron diariamente por cada uno de los tutorados, siguiendo la metodología de la reflexión en la acción de las experiencias significativas de cada día, los diarios útiles se redujeron a 137 registros, productos de ocho semanas de prácticas de seis horas diarias, con un total 240 hrs.

La instrumentación didáctica siguió una metodología organizada en tres fases:

En la primera, se llevó a cabo un taller con los tutorados durante la fase teórica de la asignatura de Fundamentos de Enfermería II, con el objeto de establecer una comunicación pedagógica entre tutoras y tutorados, se profundizó en el análisis del modelo de tutoría clínica, sus bases teórico-metodológicas, formas de participación de los tutorados y tutoras, herramientas de trabajo y específicamente se enfatizó en la metodología del diario reflexivo, funciones del tutor y tutorado, estableciendo el compromiso grupal de participación y el consentimiento informado de los tutorados acerca de su uso en el proceso de investigación.

La segunda fase, consistió en el acompañamiento de los tutorados en la práctica clínica, "El estar ahí en el escenario" donde las tutoras asesoran, dialogan y modelan prácticas específicas a cada tutorado en el servicio clínico asignado con el fin de favorecer aprendizajes significativos de los contenidos académicos. Una de las estrategias para la reflexión en la acción fue el diario reflexivo, con formato libre y con pleno respeto a la intimidad, dignidad y personalidad; diariamente debían de escribir las experiencias significativas, ${ }^{9}$ así como las reflexiones derivadas de su actuación, se les exhortó a que 
elaboraran el diario de manera personal y confidencial, asegurar que nada de lo que se escribiera en él se divulgaría con las demás compañeras, no se les calificaría, ni sus contenidos repercutirían en esta. Se acordó que los diarios se revisarían por parte del tutor, los días viernes de cada semana, de tal forma que las tutoras pudieran leerlos en el fin de semana y así reflexionar sobre las estrategias de aprendizaje a seguir con cada tutorado y hacer significativa y oportuna la tutoría clínica.

Los días lunes se establecía un encuentro entre las tutoras y los tutorados, para asesorarlos, de acuerdo a las necesidades de aprendizaje manifestadas en su diario y así propiciar la confrontación del texto para la comprensión e interpretación, además de compartir la situación personal, posterior a este encuentro se evalúa la estrategia.

La tercera fase consistió en el análisis de los textos a través de las siguientes acciones: Codificación, categorización e interpretación

Codificación, se solicitaron los diarios a los estudiantes al término de la práctica clínica, se capturan en su totalidad, fijando la atención en los datos significativos de la información, se transcriben y se establece la codificación donde se asignó un numero progresivo (01 el número del diario y CGD las iniciales del tutorado) de modo que cada diario obtuviera una ubicación única y progresiva para cada tutorado.

Posteriormente, se leyeron y se dividieron en unidades o fragmentos de texto a cada una de estas unidades que emergió de la lectura y el código de identificación luego se verificó la correspondencia entre las siglas de los códigos y los contenidos que representaban, este pro- ceso permitió detectar si existía la repetición de contenidos.

Por último, una vez que se agotó la revisión de los textos, se creó una serie de categorías derivadas de la interpretación genérica del contenido y los objetivos del estudio.

Categorización, durante este proceso se analizan los textos en los contextos se identifican algunas categorías que permiten darle sentido a las reflexiones de los tutorados, se buscan aquellos que se superponen y los significativos para la práctica, se identifican además los que deben ser eliminados. ${ }^{9,10}$

Vínculos y relaciones en el proceso de categorización, estas fueron determinadas a partir de la información de los diarios a saber: Temor Vs desconfianza, percepción sobre las enfermeras y enfermeros, identidad profesional, el ser enfermera, percepción sobre la tutoría, relación entre la teoría y la práctica.

Interpretación, estas fueron develadas a partir de los significados que le subyacen a los textos, los cuales fueron corroborados con los tutorados y comparados con los conceptos que guiaron el proceso de investigación.

La interpretación requiere de una visión hermenéutica de la totalidad de la experiencia humana que señala Dilthey acerca de la comprensión o entendimiento. A la naturaleza la explicamos, al ser humano lo comprendemos, dice el autor. "La explicación se realiza solamente a través de procesos intelectuales, pero para comprender es necesario la integración de todos los poderes mentales de la aprehensión". El acto de comprender, "implica una apertura intelectual o cognoscitiva (captación de lo objetivo), afectiva (sentir) y volitiva (querer), al complejo mundo de las experiencias humanas, pues es el ser humano entero, todo su ánimo, el que comprende". ${ }^{11}$

De esa interpretación se pueden conocer los significados que los tutorados le asignan a los procesos de aprendizaje clínico.

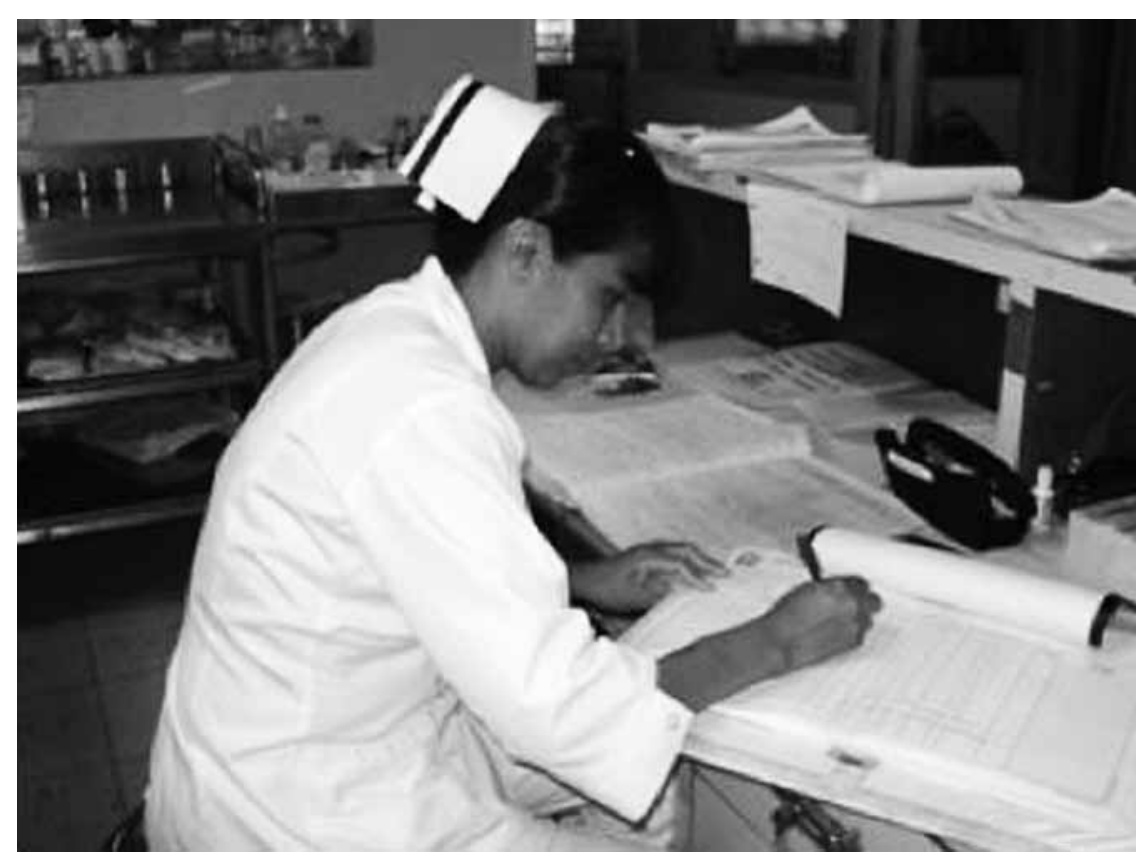




\section{Resultados}

La práctica significa el inicio de una experiencia según la cual la formación debe incluir, además de los aspectos teóricos propios del conocimiento científico y técnico, la introducción a la realidad con todas las posibilidades y dificultades que ella conlleva, es decir, el objetivo de la práctica es poner en contacto al estudiante con los diferentes ámbitos y actividades de la realidad profesional, a fin de que complete su formación asegura Calvo de León. ${ }^{12}$

Los contenidos de los diarios reflexivos son de gran variedad, unos relacionan situaciones personales y la manera de afrontamiento a la práctica; su función es darle voz al tutorado para que comparta todas las experiencias y la manera de como las vive, la forma en que las visualiza tomando en cuenta los sentimientos y emociones que en ese momento embargan su ser y que determinan su proceso de formación profesional y personal.

Con fines de confidencialidad los nombres a los que se aluden en los relatos, no son los reales y se les asignó un nombre ficticio respetando el género.

\section{Categorías de análisis}

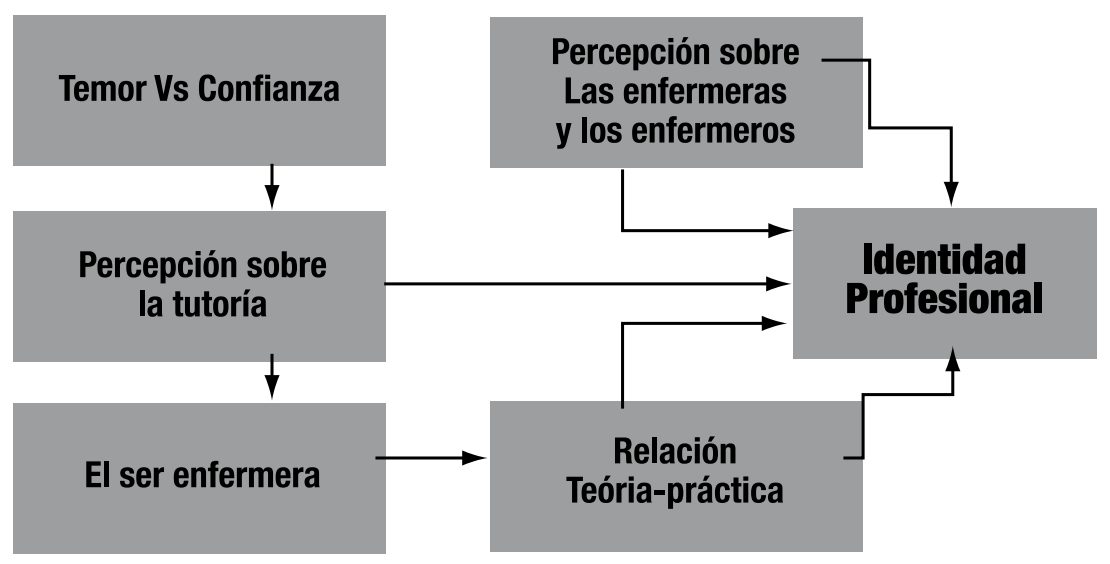

\section{Temor VS confianza}

El temor es un sentimiento común ante los cambios y situaciones nuevas que se experimentan a lo largo de la vida, se desconoce que es lo que se espera, el temor se siente en las diferentes etapas de la vida y por circunstancias variadas; es un estado afectivo, emocional, necesario para la correcta adaptación del organismo al medio, que provoca angustia en la persona.

El temor "normal" puede ser saludable hasta cierto punto, puesto que, a veces sienta las bases para el propio progreso, ayuda a preservar la vida o actúa como estímulo en el cumplimiento del deber. ${ }^{13}$

El temor es un sentimiento que se logra controlar con el pasar de los años, no en el sentido de reprimirlo y no sentirlo, pues es imposible, pero si controlarlo para que no impida continuar el camino planteado y lograr las metas, esto ocurre cuando los estudiantes de enfermería se enfrentan a un nuevo periodo de prácticas escolares, al incursionar en esta nueva etapa de la vida, ya que se tienen mayores responsabilidades y de cierto modo se continua en un proceso de maduración y crecimiento profesional. 
La confianza es la seguridad o esperanza firme que alguien tiene de otro individuo o de algo, también se trata de la presunción de uno mismo y del ánimo o vigor para obrar, es la familiaridad en el trato. Para la psicología social y la sociología, es una sensación que estima que una persona será capaz de actuar de una cierta manera frente a una determinada situación.

En este sentido, la confianza puede reforzarse o debilitarse de acuerdo a las acciones de la otra persona. La confianza supone una suspensión, al menos temporal, de la incertidumbre respecto a las acciones de los demás, cuando alguien confía en el otro, cree que puede predecir sus acciones y comportamientos. Donde hay confianza hay aumento de las posibilidades para la experiencia y la acción, hay un aumento de las posibilidades de reconciliación con la estructura porque constituye una forma más efectiva de reducción de la complejidad. La confianza, por lo tanto, simplifica las relaciones sociales. ${ }^{16}$

Los tutorados, experimentan estas situaciones ya sea al inicio de la práctica principalmente o cuando cambian de servicio, depende en gran medida de la cooperación de las enfermeras en servicio y de las tutoras cuando estas muestran actitudes de confianza y permiten que las tutoradas se incorporen al sistema social del entorno, confían en los aprendizajes previos y reflexionan de y en la práctica.

Los siguientes relatos tomados de manera textual de los diarios ya codificados permiten ver con mayor claridad las situaciones que viven los estudiantes.

"Me desperté con cierto temor, ansiedad y emoción aun no sabría
Donde hay confianza

hay aumento de las

posibilidades para la

experiencia y la acción,

hay un aumento de

las posibilidades de

reconciliación con

la estructura porque constituye una

forma más efectiva

de reducción de la complejidad.

La confianza, por lo tanto, simplifica las relaciones sociales.

cómo sería este primer día en el hospital, confieso que todo me temblaba, pero sentí más tranquilidad cuando dieron las 7:00 a.m. y ya me encontraba con mis compañeros" I-1 NB.

"Este fue nuestro primer día de práctica, estaba muy nerviosa y con miedo y sin embargo para mí fue un día regular porque tuve la suerte de que me asignaran con un buen enfermero que me preguntaba el por qué de cada procedimiento y me enseñaba" III-1 BRB.

“...me sentí un poco nervioso pues no sabía cómo me iban a recibir. Aunque todo salió bien sin contratiempos... bueno a ver qué pasa mañana" IV-2 JLSP.

"Este día como todos cuando inicio un nuevo servicio tengo nervios, pero ya en la práctica el tiempo se me fue rápido". III-5 BRB.

"Hoy estaba muy nerviosa porque inició mi práctica en el hospital. Estuve en el servicio de cirugía general, al llegar la jefa del servi- cio nos enseñó con que enfermeros íbamos a trabajar, yo con Eric y Rocío, se portaron muy amables y en la mejor disposición de enseñarme, me explicaron qué se debe de hacer al iniciar el turno: revisar los equipos, recibir pacientes, checar hojas de enfermería, checar medicamentos, armar el carrito, tomar signos vitales y ministrar medicamentos".V-2 KJBL.

"Por fin cuando conocí a los enfermeros a cargo y que lo primero que me dijeron antes de preguntar mi nombre "canaliza a esta paciente", me sentí como a prueba, pero cuando me decidí a canalizar escuché que ambos me dijeron: tu tranquila, relájate lo vas a poder hacer, creo que esas palabras me demostraron que el miedo y preocupación solo es cosa de mi cabecita loca" I-1 NB.

"Hoy por fin me sentí acoplada totalmente al servicio, ahora ya tengo mas noción del llenado de las hojas de enfermería, aunque es difícil escribir en cuadritos tan pequeños pero en fin. Hoy puse una venoclisis y se siente tan padre cuando una enfermera te llama compañera, es como si fueses alguien de tiempo en el equipo" I-3 NB.

"Hoy ya me dejaron hacer las cosas con más libertad, empecé a tomar signos vitales a mis pacientes. Conocí a otra enfermera, Adela que fue más exigente y que al igual que los otros enfermeros dispuesta a enseñarme, me iba explicando a detalle cada procedimiento, el porqué, para qué se hace y cómo se hace, al estar valorando un paciente y me enseñó con cada paciente hacer un diagnóstico de enfermería y a llenar la hoja de enfermería". V-4 KJBL. 
"Siento que si estoy aprendiendo y mejorando habilidades, creo que en equipo está muy bien porque así vas preparando los equipos y vas aprendiendo el material, las diferentes pinzas etcétera". VIII-8 MCSG.

"Hoy fue mi primer día en campo clínico, estaba muy asustada, ya que debido a una quemadura casi un mes antes no asistí a clases y no sabía nada, pero poco a poco me fui calmando". IX-2 KRM.

"Por ser mi primer día me sentía muy nervioso por no saber lo que ocurriría, en este primer día, ya que me tocaba en el servicio de medicina interna y no sabía si podría aplicar mis conocimientos o más bien si mis conocimientos serian necesarios." XII-1 AGL.

Percepción sobre las enfermeras y enfermeros

La posibilidad o potencial que las personas tienen para ir desarrollando sus habilidades depende de los demás, este potencial de desarrollo es llamado por Vygotsky Zona de desarrollo próximo. ${ }^{17}$

Desde esta perspectiva, la zona de desarrollo próximo es la posibilidad de las personas de aprender en el ambiente social, en la interacción con los demás. El conocimiento personal y la experiencia de los demás es lo que posibilita el aprendizaje; consecuentemente mientras más rica y frecuente sea la interacción con los otros, el conocimiento será más rico y amplio. En consecuencia, la zona de desarrollo próximo, está determinada socialmente, se aprende con la ayuda de los demás, en la interacción social y esta interacción como posibilidad de aprendizaje es lo que se denomina como zona de desarrollo próximo. ${ }^{17}$
En las instituciones de salud como entornos sociales donde ocurren las prácticas hospitalarias, las enfermeras y enfermeros además de las tutoras, desempeñan un papel importante en el aprendizaje de las y los tutorados, depende en gran medida de la calidad de las interacciones para que resulten significativas en su proceso de formación.

Las narrativas siguientes dan muestra de esta relación

“.. El día de hoy me encantó, porque la enfermera con la que trabajé era muy buena onda y me enseño con gusto y cortesía". III-6 BRB.

"Llegué con mucho ánimo, con ganas de trabajar y sobre todo aprender. Una vez más la enfermera de ayer es la de hoy, así que me siento en confianza". VI-2 GHD.

"El estar en ese servicio me deja una experiencia bastante buena ya que me siento bien, puesto que pude realizar bastantes cosas por la confianza que me brindaron las enfermeras que estuvieron encargadas de mí". VI-9 GHD.

“... me cuestionaban acerca de diagnósticos, padecimientos e intervenciones de enfermería además de enseñarme algunas cosas nuevas o darme tips para realizar procedimientos o interactuar con los pacientes para recabar información". VII-4 VHLG.

“...llegó la jefe de enfermeras y me dijo lo que le había comentado la enfermera del servicio, ella muy amable me preguntó cuál era el problema y yo le dije que me había puesto nerviosa pero que sabía que la presión arterial es la fuerza que ejerce la sangre sobre las paredes de las arterias; me respondió que cualquier cosa que no entendiera le preguntara a la enfermera, que es- taba en toda la disposición de ayudarme". VIII-5 MCSG.

“.... me siento muy a gusto en este servicio porque la enfermera me apoya mucho y me da la confianza de preguntarle lo que no sé". VIII-13MCSG.

"Me la pasé súper y aprendí mucho hoy, además....las enfermeras, me dijeron que me podían ayudar a aprender sobre los demás servicios del hospital, ya estoy mucho más relajada con respecto a mi práctica". IX-5 KRM.

"La enfermera que me tocó para ser sincera no me explicaba muchas cosas y no le veía mucho el interés de enseñarme". X-10 MMMM.

"Hoy llegó el otro compañero del enfermero Cesar, Miguel Ángel que es el auxiliar asignado a trabajar con él, la verdad sabe mucho y es un buen maestro, además de que juntos son un completo relajo, bromean mucho entre ellos y hasta con los pacientes, claro sin faltarles el respeto, a mí eso me parece muy bien, los hacen reír y hacen que su estancia en el hospital sea menos tediosa". XI-7 AGG.

"Este servicio me encantó ya que el enfermero Rubén, me da mucha confianza y me deja hacer todos los procedimientos, si me equivoco en algo solo me pide que pare y que analice que estoy haciendo mal y porqué, no me regaña ni se enoja, al contrario me motiva y me pide que estudie lo relacionado a los diagnósticos de enfermería de los pacientes". XIII-8 JCVB.

\section{Identidad profesional}

La relación que guarda la construcción de la identidad personal con la profesión de enfermería es que esta construcción se da también en un contexto sociocultural. 
Durante la vida estudiantil, los jóvenes heredan un rol tradicional asignado por la sociedad que consiste en prolongar el cuidado y el servicio a los demás que se realiza en la casa hacia el hospital. Las tutoras de enfermería, las enfermeras y enfermeros en servicio y todos aquellos profesionistas que intervienen en el proceso educativo de los alumnos, participan en la construcción de esa identidad como enfermeras y hacia la enfermería. Si esa construcción fortalece la seguridad personal, la autoestima y el auto-concepto del alumno, esa identidad con la enfermería será positiva. ${ }^{18}$

Las siguientes narrativas ilustran la identidad profesional que se está desarrollando en los tutorados:

"Una paciente me preguntó ¿porque estas estudiando enfermería? me movió mucho porque me hizo recordar sucesos familiares muy dolorosos que tienen que ver con su pregunta y pues le dije que me ha gustado siempre todo lo relacionado al cuerpo humano, yo tuve una hermana que falleció de IRC lo cual me hizo dudar si yo quería seguir adelante, le dije que ahora cada vez que veo a un paciente, trato de ser lo más atenta y llevar a cabo mis procedimientos, de la mejor forma posible, ya que eso me hace sentir muy bien porque les doy la atención y cuidados que hubiera querido para mi hermana y que también ha sido difícil, pero que aun así me gustaría especializarme en cardiología, ya que desde que conocí las funciones del corazón me ha llamado mucho la atención". V-5 KJBL.

"Conocí a un paciente que me dijo unas sabias palabras que no olvidare si te gusta mucho la ca- rrera o cualquier otra si estudias y te preparas serás la mejor, supérate y gracias por toda la atención recibida.V-9 KJBL.

“...... escuché que se iba ese mismo día de alta, me llenó de gusto, alegría satisfacción fue un manojo de emociones las que llegaron a mí en ese momento, me despedí diciéndole a la señora, que siempre la iba a recordar, pues gracias a ella lejos de aprender procedimientos y demás pude ver que esta es mi verdadera profesión". VII-6 VHLG.

"Creo que ya le estoy perdiendo el miedo a los pacientes y me gusta ayudar a las personas, es muy gratificante una sonrisa y un "gracias" y creo que en ocasiones que nosotros como estudiantes somos más atentos" VIII-6 MCSG.

"Me despedí de ellos y me desearon buena suerte, me voy muy satisfecha de mis prácticas particularmente de este servicio, porque al convivir con este tipo de pacientes que no pueden ver y que a pesar de eso le echen ganas y sigan con ese ánimo de vivir, me dejan una enseñanza muy padre de que pase lo que pase no hay que dejarse vencer por nada". VIII-19MCSG.

"Doña Lulú y don Ricardo me ayudaron indirectamente a reafirmar la elección de mi carrera, es algo muy padre e inexplicable lo que ahora siento, el saber que puedo ayudar a una persona y que ellos me lo agradezcan, dar calidad de cuidado y no cantidad; lo que me sirve es ponerme en el lugar de la otra persona o lo que se llama ser empático, para poder entender mejor a la persona IX-5 KRG.

"Yo no quería estudiar enfermería, pero estos cuatro días y en especial hoy me hicieron darme cuenta que esto me gustaba. No se compara con que el paciente te de gracias y te felicite por tu trabajo y saber que de algo les ayudaste para sobre llevar su enfermedad" XI-9 AGG.

\section{El ser enfermera}

Todo cuidado de enfermería reconoce al ser persona tanto a la enfermera como al paciente, implica un acto de consciencia, es decir, es intencionado, no se da espontáneo, la enfermera debe querer cuidar a las personas como un acto ético, es cuidar intencionalmente, implica tener consciencia de que la manera en que la enfermera se percibe a sí misma afecta la manera en que percibe a los otros. Por lo tanto, involucra querer conocerse a sí misma cada día, querer darse cuenta que ella y la persona cuidada se encuentran en los mismos procesos del vivir que implican cambios y transformaciones, como es la maduración y el envejecimiento, con sus consecuencias psicológicas y sociales, con la conciencia de la propia muerte y la angustia ante la posibilidad de la destrucción del propio ser, o el dolor ante la pérdida de alguien que amamos, que es parte de nosotros. Y todo esto lo comparte la enfermera con su paciente, porque ambos comparten la misma humanidad.

La enfermera persona que cuida al otro se reconoce persona, ya que de otra manera no es posible encontrarse con el otro, pues para hacerlo es necesario vaciarse temporalmente del juicio racional sobre el otro, para poder acogerlo en su singularidad y escuchar con atención para comprender la experiencia total del otro y podrá acompañarlo en sus transiciones. Necesita también conocerse comprenderse, 
aceptarse y actualizarse. Entonces, esta relación de cuidado se lleva a cabo mediante un encuentro de cuidado persona a persona, en el que la enfermera percibe poco a poco al otro como tú, quien, desde la concepción de Buber, ${ }^{19}$ se va develando como ser, en sus valores y significados.

Es así que las y los tutorados experimentan este proceso de ser enfermeras en la interacción con las personas que cuidan como se evidencia en los siguientes relatos:

"Como a las 11:00 a.m. me dijeron que si quería amortajar a un paciente y lo hice sin ningún miedo, ni pudor pero si con mucho respeto. En mi mente le enviaba buenos pensamientos de acuerdo a mis creencias". VI-2 GHD.

"...teníamos mas contacto con los pacientes y me agrado mucho". VII-3 VHLG.

“...ese mismo día le di de comer a la señora Refugio la cual tiene dificultades visuales, me di cuenta, que se llenaron de agua sus ojos le pregunté qué era lo que tenía, tristemente contestó que estaba llorando y rompió en llanto, en ese momento sentí un gran nudo en la garganta pero me tuve que aguantar, traté de consolar a la señora, después de unos instantes terminó de llorar y empezó a comer (y eso porque la convencí) terminada mi tarea continué con las demás personas". VII-5 VHLG

"Sin darme cuenta le tomé cariño a la señora Refugio pues me tocara o no me tocara atenderla yo estaba al pendiente de ella, y lo que recibí a cambio fueron bendiciones pero no solo de ella sino de todas las señoras que cuidé, para muchos pueden ser solo palabras, pero para mí significaron uno de los regalos más gratos en mi vida". VII-5 VHLG

“...me llevo experiencias y amistades muy buenas y también algunas malas, pero dentro de todo hay que aprender a ser éticos y humanos". VII-7 VHLG.

“...cuando me la encontré en la mañana le pregunte que si que si ya la iban a operar y me dijo que si que la cirugía estaba programada a las 9:00 a.m. y me dijo "me llamo Lety, rezas por mi" y le dije si señora ya verá que saldrá bien, cuando llegaron por ella los camilleros yo estuve ahí y la acompañe hasta el quirófano y me tomó la mano y me sonrió y sentí muy bonito". VIII-1 MCSG

"...tercer semestre era crucial para saber si esto era lo que queríamos hacer y así fue, tomé la decisión correcta, estoy emocionada y enamorada de la enfermería iiMUCHAS GRACIAS!! ENEO." VIII-19 MCSG.

"Me siento feliz porque creía que no podía sola y pude, salí adelante. Aprendí mucho, hoy me siento muy orgullosa de mi". IX-6 KRM.

"El día de hoy estuvo muy tranquilo en urgencias y hoy sentí bonito porque al ayudar a una persona mayor, ella me dijo perdón por darle tantas molestias, a lo cual yo dije que no era ninguna que para eso estábamos y me sentí muy bien porque me di cuenta que las personas dependen de nosotras y tu estas ahí para ayudarlas". X-8 MMM.

"Una señora que lleva por nombre María de Jesús me puse a platicar ya que la vi muy preocupada, ella dijo que era por su familia y sus hijos, trate de calmarla y de darle una terapia afectiva que la tranquilizara". X-19 MMM.

"Ellos de igual manera me desearon lo mejor y me dieron las gracias por la atención que les di. Eso me hizo sentir satisfecha conmigo misma, pero a la vez tristeza porque ya no los iba a ver". X-22 MMM.

\section{Percepción sobre la tutoría.}

Esta concepción se introduce recientemente en la enseñanza aprendizaje de la enfermería clínica, en vez de la supervisión de la práctica, superando una concepción mecanicista del quehacer docente, así se conceptualiza como "Un proceso de acompañamiento colaborativo a través de la atención personalizada a un tutorado o grupo reducido, por tutores expertos basados en el aprendizaje práctico, estrategias pedagógicas reflexivas y una comunicación dialógica, que permita atender las necesidades del tutorado para su formación profesional integral, donde tutor y tutorado se comprometen en el aprendizaje del cuidado". 3

La acción tutorial involucra al tutor y tutorado en la práctica, esto implica estar ahí en el escenario, considerando todas las experiencias, interacciones y asunción de los roles, de acuerdo a las necesidades del entorno hospitalario, como campo de práctica de la enfermería. Un ejemplo de ello son las siguientes expresiones de los tutorados:

"Este día me dio muchísimo gusto estar en el programa de tutoría, por que las cosas tan básicas que ahí ocurren, como preparar el material, no lo sabía, en mi vida me hubiera imaginado que fuera así". III-2. BRB

"Muchas gracias a las tutoras A y $B$ por la entrevista tan chida que me realizó y a la $C$, que siempre estuvieron con nosotros".III-5 BRB. 
"Se me olvidó comentarte (diario) que eres parte de mi proceso de aprendizaje y por ello, mis vivencias serán leídas por mis tutoras. Ellas te cuidaran cuando no estés conmigo". IX-6 KRM.

"...nos fuimos con los de $5^{\circ}$. que son como otra fuente de información, nos preguntan y nos resuelven dudas, es bueno contar con gente así. Hasta da gusto preguntar así, tanto al personal como a compañeros de otros semestres, nos tienen mucha paciencia y cada día nos dan algo nuevo". XI-3 AGG.

"Al poco rato llegó la tutora y más nerviosa me puse, pensé que a lo mejor ella también me regañaría, pero la verdad fue todo lo contrario, a lo que pensé, pues me apoyo y me tranquilice". XI-5 AGG.

"De hecho ya tenía un estudiante del IMSS, Alma, cuando supe que tenía que trabajar con ella solo esperé que no hubiera rencillas entre nosotras por ser de diferente escuela, pero fue todo lo contrario ella y trabajamos muy bien. Hasta estuvo bien que me tocara con ella sabía mucho y aprendí mucho de ella, fue un buen día", XI-6 AGG.

"Al final de la semana me sentí mucho mejor, porque me voy familiarizando con los servicios, además de que voy adquiriendo mayor práctica y la presión que sentía al principio se va perdiendo, gracias al apoyo de las enfermeras, mi profesora y las tutoras que siempre están al pendiente de nosotros". XIII7 JCVB.

\section{Relación entre la teoría y la práctica.}

La conexión entre la teoría y práctica contribuye a la formación integral del individuo, ya que a través de esta relación es posible que el futuro profesional aprenda a desenvolverse en la acción, es decir, en los acontecimientos cotidianos, sobre los cuales actuará una vez finalizada su formación. ${ }^{8}$

También señala Schön, ${ }^{8}$ la necesidad de replantear la epistemología de la práctica, pues ante los problemas que esta presenta, los profesionales apelan a la racionalidad técnica, la cual "defiende la idea de que los profesionales de la práctica solucionan problemas instrumentales, mediante la selección de los medios técnicos más idóneos para determinados propósitos", ${ }^{8}$ subraya las limitaciones de la racionalidad técnica, en cuanto no posibilita la resolución de problemas tales, como las situaciones de incertidumbre, el tratamiento de lo singular o la toma de decisiones axiológicas. Los siguientes relatos dan cuenta de esta relación.

La conexión entre

la teoría y práctica contribuye a la formación integral del individuo, ya que a través de esta relación es posible que el futuro profesional aprenda a desenvolverse en la acción, es decir, en los acontecimientos cotidianos, sobre los cuales actuará una vez finalizada su formación.
"Lo único malo de ese día fue que me di cuenta, de que todos los procedimientos que nos enseñaron en la escuela, en el hospital se hace todo lo contrario y es aquí en donde tengo una confusión, de cómo hacerlos, ¿cómo me enseñaron en la escuela o en el hospital?.." III -1 BRB.

"Y que desilusión que no se amortaja como me enseñaron en la escuela, es más, casi nada se hace como en la escuela. Ahora sí que estoy confundida" Diario VI-2 GHD.

"Me incorporé al servicio de medicina interna en donde se encuentra el de Diálisis; preparamos el carrito, tomamos signos vitales pero la enfermera me dijo que tenía que tomar el pulso y la frecuencia respiratoria quince segundos y multiplicarlo por cuatro, que así se tenía que hacer". VIII-4 MCSG.

\section{Conclusiones}

Con la implementación del proyecto de la tutoría clínica en la práctica, se comprueba que el diario reflexivo, es una herramienta de enseñanza aprendizaje que favorece el aprendizaje reflexivo y permite reconocer situaciones personales y grupales de los tutorados que intervienen en el proceso de aprender; a su vez permite mantener una relación tutor-tutorado más estrecha, favorece el dialogo acerca de los fenómenos del cuidado, el tutorado adquiere seguridad en la realización de procesos clínicos. Esta relación va aumentando el nivel de confianza y seguridad en los tutorados, como se visualiza en los relatos de los diarios reflexivos.

Los diarios reflexivos develan las áreas de aprendizaje que requieren ser fundamentados duran- 
te la práctica, así como las áreas y situaciones en las que se tiene que reflexionar, esta actividad intencionada permite mejorar el aprendizaje de la Enfermería Fundamental.

Como instrumento de aprendizaje permite individualizar la tutoría tanto en necesidades académicas como personales y profesionales. La reflexión en la acción coloca al tutorado-tutor en un nivel metodológico propicio para la tutoría.

La acción tutorial permite repensar la vinculación de la teoríapráctica desde otra dimensión ya que implica una relación dialéctica, donde el punto de partida es la práctica para reflexionar y teorizar sobre ella y volver a la acción, lo que Schön denomina el prácticum reflexivo. ${ }^{20}$

Encontrar los significados que los tutorados le asignan a su práctica de enfermería es un proceso necesario para la reflexión de la acción como una metodología en el aprendizaje del cuidado y el análisis de los diarios reflexivos dan cuenta de los procesos que el tutorado atraviesa durante su experiencia clínica, a partir de ellos el tutor interviene de manera concreta según sean las necesidades del tutorado y se ve fortalecida la relación pedagógica entre tutor-tutorado.

Como estrategia de aprendizaje el diario reflexivo se puede implementar vinculado a un proyecto integral de tutoría clínica que junto con la intervención pedagógica del tutor, las profesionales de enfermería, la acción reflexiva de los tutorados y el programa académico potencialicen el desarrollo de las habilidades, los conocimientos y los valores profesionales deseables para una formación integral.

\section{Referencias Bibliográficas}

1 Miles MB, Huberman AM. Qualitative data analysis: Anexpanded sourcebook. 2 ed. Thousand Oaks, CA: Sage Publications, Inc. 1994. p. $35-42$

2 Cunha MI. Conta-me agora: as narrativas como alternativas pedagógicas na pesquisa e no ensino. Rev da Fac de Educ [Internet]. 2005 [acceso 18 de abril de 2012]; 23: [23-28]. Disponible en: http://bit. ly/Y2TYKF

3 Rodríguez-Jiménez S, Cárdenas JM. La tutoría clínica reflexiva: Un modelo de práctica docente en enfermería. México: UNAM, Porrúa; 2011. p. 83

4 Bradbury-Jones C. Enhancing rigour in qualitative health research: exploring subjectivity through Peshkin's I's. Journal of Advanced Nursing. 2007; 59 (3): 290-298.

5 Mayorga CF, Friedeman Ml, Jiménez LD, Díaz L. Diarios personales del investigador como herramienta de investigación. Av. Enferm [Internet]. 2008 [acceso 20 de abril de 2012]; 26 (2): [15-26]. Disponible en: http://bit.ly/XJwISs

6 Bourdieu P. La distinción. Criterios y bases sociales del gusto. Madrid: Taurus; 1988. p. 170-171.

7 Quevedo-Marcolino T. El trabajo del mentor. Análisis de los feedbacks de diarios reflexivos a lo largo de un proceso de mentoría en grupo. Rev. Ibero de Educ. XX1 [Internet]. 2012 [acceso 28 abril de 2012]; 52 (6): [93-118]. Disponible en: http://bit.ly/VQXBqh

8 Schön D. La formación de profesionales reflexivos: Hacia un nuevo diseño de la Enseñanza y el Aprendizaje en las profesiones. Barcelona: Paidós; 1992.

9 Taylor SJ. y Bodgan R. Introducción a los métodos cualitativos de inves- tigación. México: Paidós; 1986. p. 15-27

10 Chacón-Corzo MA. Estrategias de enseñanza reflexiva en la formación inicial docente Educere 2008; (41): 277-287. Disponible en: http://bit.ly/15UshuE

11 Dilthey W. La esencia de la filosofía. Buenos Aires, Arg: Losada; 1944.

12 Calvo de León R, Esteban-Ruiz F. El prácticum en la formación de educadores sociales. XIV Seminario interuniversitario de Pedagogía Social. Universidad de Burgos [internet] 1999. Disponible en: http:// bit.ly/WuxEda

13 Abarca N. Inteligencia emocional en el liderazgo, Santiago: El Mercurio-Aguilar; 2009. p.58

14 Kramer RM. Repensar la confianza. Harvard Business Review. 2009; 87 (6): $73-82$.

15 Solomon R, Flores F. Building Trust: in Business Politics, Relationships, and Life. Nueva York: Oxford University Press; 2001.

1616 Luhmann N. Confianza. México: Anthropos; 1996.

17 Casanueva-Sáez P. Evaluación Educacional Formadora. Monog [Internet]; 2005. Disponible en: http://bit.ly/XoBPcO

18 Loo I. La identidad como proceso biológico-psicosocial. Rev Enferm Inst Mex Seguro Soc 2003; 11 (1): 49-54.

19 Buber M. ¿Qué es el hombre?. México, FCE. 1976.

20 Ochoa de Rigual N, Rodríguez-Noriega Y. La teoría de la acción reflexiva: algo para reflexionar mientras se acciona. Revista Ciencias de la Educación 2005; 2 (26): 225-233. 